\title{
Effect of dapagliflozin on obstructive sleep apnea in patients with type 2 diabetes: a preliminary study
}

Yi Tang ${ }^{1}$, Qin Sun ${ }^{2}$, Xiao-Yan Bai ${ }^{3}$, Yun-Fan Zhou', Qiong-Lan Zhou ${ }^{5}$ and Min Zhang ${ }^{2}$

\begin{abstract}
Objective: The aim of this case-control study was to assess the efficacy of dapagliflozin combined with metformin for type-2 diabetes mellitus (T2DM) with obstructive sleep apnea hypopnea syndrome (OSAHS).

Methods: A total of 36 patients with newly-diagnosed T2DM and OSAHS were randomized divided into two groups. Eighteen OSAHS patients with T2DM, who were treated with dapagliflozin and metformin, were assigned as the dapagliflozin group. These patients were given dapagliflozin and metformin for 24 weeks between February 2017 and February 2018. Another 18 OSAHS patients with T2DM, who were treated with glimepiride and metformin for 24 weeks, were assigned as the control group. Fasting plasma glucose (FPG) level, postprandial blood glucose (PPG), hemoglobin A1C (HbA1c), fasting insulin, homeostasis model assessment of insulin resistance (HOMA-IR), blood lipids, body mass index (BMI), blood pressure, apnea-hypopnea index (AHI), minimum oxygen saturation $\left(\mathrm{LSpO}_{2}\right)$, and Epworth Somnolence Scale (ESS) score were measured before and at 24 weeks after the initiation of treatment.

Results: In the dapagliflozin group, triglyceride (TG), systolic pressure (SBP) and diastolic pressure (DBP) significantly decreased following treatment, while high-density lipoprotein cholesterol $(H D L-C)$ significantly increased $(P<0.05)$. Furthermore, a reduction in $\mathrm{AHI}$, an increase in $\mathrm{LSpO}_{2}$ and a decrease in ESS score were observed in the dapagliflozin group $(P<0.05)$, but not in the control group. Moreover, blood glucose, HbA1c, HOMA-IR, and BMI significantly decreased in these two groups, and the decrease was more significant in the dapagliflozin group.

Conclusion: These present results indicate that dapagliflozin can significantly reduce glucose, BMl, blood pressure and $\mathrm{AHI}$, and improve hypoxemia during sleep and excessive daytime sleepiness, which thereby has potential as an effective treatment approach for OSAHS.
\end{abstract}

\section{Introduction}

Obstructive sleep apnea hypopnea syndrome (OSAHS) includes a constellation of symptoms characterized by total obstruction (apnea) or partial obstruction

\footnotetext{
Correspondence: Qin Sun (sunqin1947@163.com) or

Min Zhang (zhangmcd@163.com)

${ }^{1}$ Department of Endocrinology, The Fifth People's Hospital of Chengdu, 611130 Chengdu, P.R. China

${ }^{2}$ Center of Diabetes Mellitus, Sichuan Academy of Medical Sciences and

Sichuan Provincial People's Hospital, 610072 Chengdu, P. R. China

Full list of author information is available at the end of the article.

These authors contributed equally: Yi Tang, Qin Sun
}

(hypopnea) of the upper airways during sleep ${ }^{1}$. Patients with OSAHS have apnea or reduced breathing during sleep, which causes anoxia, carbon dioxide retention, nocturnal sleep apnea, and excessive daytime sleepiness (EDS). Furthermore, OSAHS can induce cognitive decline and autonomic modulation disorder. In addition, OSAHS is closely correlated to obesity, type- 2 diabetes mellitus (T2DM) and cardiovascular diseases ${ }^{2,3}$, and $60-70 \%$ of OSAHS patients are overweight or obese. The prevalence of OSAHS in the T2DM patients with obesity is $86 \%{ }^{4}$. Untreated moderate and severe OSAHS is considered to 
be a key risk factor for cardiovascular events and death ${ }^{5-7}$. Many patients suffer from obesity. Clinical practice guidelines recommend that overweight adult OSAHS patients should reduce weight, because weight loss helps improve ventilation and prognosis in these patients ${ }^{8,9}$.

A new hypoglycemic drug, dapagliflozin, which is a kind of sodium glucose co-transporter 2 (SGLT2) inhibitor, can reduce the reabsorption of glucose by the kidney, increases urine glucose excretion, and reduce blood glucose levels in T2DM patients ${ }^{10,11}$. It has also been found that this inhibitor can reduce weight, lower blood pressure, and improve hyperlipidemia ${ }^{12-14}$. Another classic hypoglycemic drug, metformin, has been proven to benefit weight loss in T2DM patients ${ }^{15,16}$.

Dapagliflozin and metformin can effectively reduce the body weight of patients with OSAHS ${ }^{17}$. Therefore, it is assumed that these might have potential effects in improving the symptoms, and even the breathing of OSAHS patients. In order to verify this idea, a randomized-controlled trial was designed to observe the effects of metformin combined with dapagliflozin on body mass index (BMI), and the breathing and sleep quality of patients with OSAHS combined with T2DM.

\section{Materials and methods Patients}

A total of 36 patients with newly diagnosed T2DM and OSAHS were included in the study cohort. These patients were enrolled from the Fifth People's Hospital of Chengdu and Sichuan Provincial People's Hospital between February 2017 and February 2018. The diagnose of OSAHS was completed based on symptoms, such as snoring, respiratory arrest, suffocation, daytime drowsiness, fatigue, lack of energy and the relevant inspection. The age of these participants ranged within $18-65$ years old, and their $\mathrm{HbA}_{1 \mathrm{C}}$ was $>7.5 \%$, but $<9 \%$. All these participants could not effectively control their blood glucose when using the largest dose of metformin, and needed other hypoglycemic drugs. Patients with moderate-severe kidney failure and liver damage were excluded to decrease the onset of oral antidiabetic drug-related side effects. Patients with serious lung function decline, neuromuscular diseases, and congestive heart failure, as well as patients who recently had acute infection and surgery, were also excluded. All patients were recommended to adopt the routine diet and exercise regimen for patients with diabetes.

A total of 36 patients were divided into two groups: dapagliflozin group $(n=18)$, and control group $(n=18)$. The randomization was performed by a computergenerated number. Allocation was issued using opaque, sealed, numbered envelopes. The sample size was calculated by the statistical software. The dose of the hypoglycemic drug was regulated according to the blood sugar in the whole trial. These two groups were required to receive a large dose of metformin $(0.85 \mathrm{~g}$ bid). All 18 patients in the dapagliflozin group received $5 \mathrm{mg}$ of dapagliflozin every day, and $10 \mathrm{mg}$ was added one week later, for a total of 24 weeks. The 18 subjects in the control group received $2 \mathrm{mg}$ of glimepiride every day. If the glucose of these patients were not well-managed, this could be increased to $4 \mathrm{mg} /$ day. The treatment duration also lasted for 24 weeks.

The present study was approved by the ethics committees of the Fifth People's Hospital of Chengdu and Sichuan Provincial People's Hospital, and both committees provided a waiver of consent. All participants had signed the informed consent.

\section{Evaluations}

The primary outcome was glucose level, including fasting plasma glucose (FPG) level, and postprandial blood glucose (PPG). Besides, hemoglobin A1C (HbA1c), fasting insulin, homeostasis model assessment of insulin resistance (HOMA-IR), blood lipids, BMI, blood pressure, apnea-hypopnea index (AHI), and minimum oxygen saturation $\left(\mathrm{LSpO}_{2}\right)$ were also detected, and the severity of daytime sleepiness was evaluated using the Epworth Somnolence Scale (ESS) score. Blood glucose and HbA1c was respectively determined using the glucose oxidase method and high performance liquid chromatography. FPG, PPG, HbA1c, fasting insulin and blood lipids were detected by ANAYTECH-640 automatic biochemical analysis. AHI and $\mathrm{LSpO}_{2}$ were measured using a portabletype sleep apnea detector (JF-1000, Beijing YiheJiaye Medical Technology Corporation). The above indexes were reviewed after 24 weeks.

\section{Statistical analysis}

SPSS 23.0 statistical software was used to statistically analyze the clinical data. Measurement data were expressed with mean \pm standard deviation. The normally distributed continuous data were compared using independent $t$-test, while the non-normally distributed continuous data were compared using non-parametric tests. The count data were analyzed using chi-square test. If $P<$ 0.05 , the difference between groups was considered to be statistically significant.

\section{Results}

\section{Patient characteristics}

Table 1 presents the characteristics of the 36 patients with OSAHS and T2DM before the follow-up. There were no significant differences in clinical profiles between the dapagliflozin group and control group.

\section{Changes in clinical and laboratory findings}

The changes in clinical and laboratory findings for the dapagliflozin and control groups are presented in Table 2. 
Table 1 Clinical characteristics at start of follow up

\begin{tabular}{llll}
\hline & Dapagliflozin $(\boldsymbol{n}=\mathbf{1 8})$ & Control $(\boldsymbol{n}=\mathbf{1 8})$ & $\boldsymbol{P}$-vaule \\
\hline Age & $56.10 \pm 7.21$ & $57.88 \pm 10.07$ & 0.813 \\
Male (\%) & $55 \%$ & $65 \%$ & 0.519 \\
History & $1.12 \pm 0.30$ & $1.15 \pm 0.26$ & 0.742 \\
BMI (kg/m $\left.{ }^{2}\right)$ & $28.17 \pm 1.21$ & $28.04 \pm 1.19$ & 0.746 \\
FPG (mmol/L) & $8.24 \pm 0.29$ & $8.12 \pm 0.47$ & 0.360 \\
PPG (mmol/L) & $14.86 \pm 1.58$ & $14.76 \pm 1.63$ & 0.861 \\
HbA1c (\%) & $8.38 \pm 0.95$ & $8.45 \pm 1.10$ & 0.830 \\
HOMR-IR & $4.03 \pm 0.31$ & $4.06 \pm 0.33$ & 0.786 \\
TG (mmol/L) & $1.85 \pm 0.47$ & $1.89 \pm 0.40$ & 0.824 \\
LDL (mmol/L) & $2.79 \pm 0.32$ & $2.67 \pm 0.49$ & 0.907 \\
HDL (mmol/L) & $0.96 \pm 0.13$ & $0.95 \pm 0.14$ & 0.941 \\
SBP (mmHg) & $151.22 \pm 5.95$ & $152.56 \pm 7.25$ & 0.551 \\
DBP (mmHg) & $92.44 \pm 7.62$ & $93.16 \pm 6.23$ & 0.887 \\
AHI(events/h) & $37.45 \pm 6.04$ & $38.11 \pm 6.27$ & 0.747 \\
LSp O2 (\%) & $84.06 \pm 14.58$ & $83.72 \pm 13.77$ & 0.812 \\
ESS score & $11.83 \pm 3.76$ & $12.50 \pm 3.79$ & 0.600 \\
\hline
\end{tabular}

TG Triglyceride, $L D L$ low density lipoprotein cholesterol, $H D L$ high density lipoprotein cholesterol, $S B P$ systolic pressure, $D B P$ diastolic pressure

Table 2 Changes of clinical and laboratory findings after treatment

\begin{tabular}{|c|c|c|c|c|c|c|c|c|}
\hline & \multicolumn{3}{|c|}{ Dapagliflozin ( $n=18$ ) } & \multicolumn{3}{|c|}{ Controls $(n=18)$} & \multicolumn{2}{|l|}{ Difference } \\
\hline & Baseline & Posttreatment & P-vaule & Baseline & Posttreatment & $P$-vaule & Mean & $P$-vaule \\
\hline BMI $\left(\mathrm{kg} / \mathrm{m}^{2}\right)$ & $28.17 \pm 1.21$ & $25.92 \pm 0.92$ & 0.000 & $28.04 \pm 1.19$ & $27.04 \pm 1.11$ & 0.000 & $-1.21 \pm 0.85$ & 0.004 \\
\hline FPG (mmol/L) & $8.24 \pm 0.29$ & $6.56 \pm 0.38$ & 0.000 & $8.12 \pm 0.47$ & $6.53 \pm 0.32$ & 0.000 & $0.02 \pm 0.28$ & 0.850 \\
\hline PPG (mmol/L) & $14.86 \pm 1.58$ & $9.95 \pm 1.62$ & 0.000 & $14.76 \pm 1.63$ & $10.23 \pm 0.95$ & 0.000 & $-0.21 \pm 1.40$ & 0.563 \\
\hline HbA1c (\%) & $8.38 \pm 0.95$ & $6.49 \pm 0.71$ & 0.000 & $8.45 \pm 1.10$ & $6.35 \pm 0.36$ & 0.000 & $0.18 \pm 0.75$ & 0.473 \\
\hline HOMR-IR & $4.03 \pm 0.31$ & $3.07 \pm 0.27$ & 0.000 & $4.06 \pm 0.33$ & $3.94 \pm 0.25$ & 0.010 & $-0.79 \pm 0.49$ & 0.000 \\
\hline TG (mmol/L) & $1.85 \pm 0.47$ & $1.58 \pm 0.51$ & 0.004 & $1.89 \pm 0.40$ & $1.86 \pm 0.38$ & 0.220 & $-0.28 \pm 0.31$ & 0.028 \\
\hline LDL (mmol/L) & $2.79 \pm 0.32$ & $2.77 \pm 0.28$ & 0.174 & $2.67 \pm 0.49$ & $2.61 \pm 0.34$ & 0.301 & $0.15 \pm 0.51$ & 0.172 \\
\hline $\mathrm{HDL}(\mathrm{mmol} / \mathrm{L})$ & $0.96 \pm 0.13$ & $1.06 \pm 0.12$ & 0.001 & $0.95 \pm 0.14$ & $0.96 \pm 0.13$ & 0.084 & $0.09 \pm 0.14$ & 0.036 \\
\hline $\mathrm{SBP}(\mathrm{mmHg})$ & $151.22 \pm 5.95$ & $146.11 \pm 6.45$ & 0.000 & $152.56 \pm 7.25$ & $150.25 \pm 7.38$ & 0.725 & $-6.11 \pm 6.63$ & 0.012 \\
\hline $\mathrm{DBP}(\mathrm{mmHg})$ & $92.44 \pm 7.62$ & $86.33 \pm 7.17$ & 0.000 & $93.16 \pm 6.23$ & $92.12 \pm 7.16$ & 0.907 & $-5.88 \pm 8.56$ & 0.019 \\
\hline AHI (events/h) & $37.45 \pm 6.04$ & $26.72 \pm 4.69$ & 0.000 & $38.11 \pm 6.27$ & $36.1 \pm 4.50$ & 0.265 & $-10.17 \pm 6.96$ & 0.000 \\
\hline LSp O2 (\%) & $84.06 \pm 14.58$ & $87.16 \pm 13.56$ & 0.004 & $83.72 \pm 13.77$ & $84.12 \pm 13.83$ & 0.479 & $3.00 \pm 4.46$ & 0.020 \\
\hline ESSscore & $11.83 \pm 3.76$ & $9.05 \pm 2.75$ & 0.001 & $12.50 \pm 3.79$ & $12.11 \pm 3.16$ & 0.422 & $-1.78 \pm 2.16$ & 0.004 \\
\hline
\end{tabular}

TG triglyceride, $L D L$ low density lipoprotein cholesterol, $H D L$ high density lipoprotein cholesterol, SBP systolic pressure, $D B P$ diastolic pressure

After 24 weeks of treatment, FPG, PPG, HbA1c, HOMAIR, and BMI significantly decreased in these two groups $(P<0.05)$. Compared with the control group, HOMA-IR and BMI significantly decreased in the dapagliflozin group $(P<0.05)$, while FPG, PPG, and HbA1c did not decrease $(P>0.05)$. The glucose levels in these two groups were all effectively managed. Compared to the control group, and although the regimen of dapagliflozin 
combined with metformin had no significant advantage in reducing glucose, the treatment scheme could be more effective in improving the patient's insulin resistance, and reducing the patient's weight.

In the dapagliflozin group, TG, SBP, and DBP significantly decreased after the treatment, while HDL-C significantly increased $(P<0.05)$. The significant changes of these characteristics were not observed in the control group.

A reduction in AHI, an increase in $\mathrm{LSpO}_{2}$, and a decrease in ESS score were observed in the dapagliflozin group $(P<0.05)$, but not in the control group, indicating the amelioration of breathing and EDS after the administration of dapagliflozin and metformin.

\section{Discussion}

The present study found that the combination of dapagliflozin and metformin not only alleviated the patient's weight, and improved the blood glucose, blood pressure, and blood lipid levels of patients with T2DM and OSAHS, but also significantly improved the patient's ventilation and daytime sleepiness, showing that the symptoms of OSAHS patients were ameliorated. Previous studies have pointed out that metformin can reduce the weight of T2DM and OSAHS patients, but it did not enhance the ventilatory function ${ }^{18}$ in these patients, and did not appear to be able to prevent the development process ${ }^{19}$. Therefore, it was considered that dapagliflozin may have played a major role in improving ventilation in patients with T2DM and OSAHS.

Although the improvement of OSAHS is closely correlated to the degree of weight loss, the mechanism for improvement in $\mathrm{AHI}$ and $\mathrm{LSpO}_{2}$ by dapagliflozin in the present study remains unclear. Weight loss maybe one reason, but the change in body composition may have been more important than the reduction in $\mathrm{BMI}^{20-22}$. Several researches have suggested that diuretic therapy can cause a decrease in AHI in patients with OSAHS, without a marked reduction in body weight ${ }^{23-26}$. Furthermore, researchers have inferred that diuretics lead to an overnight fluid shift from the legs to the neck during sleep $^{27}$, with this redistribution partly contributes to the severity of obstructive sleep apnea. Moreover, dapagliflozin has diuretic effects, and changes the body composition $^{28,29}$.

EDS is an important clinical feature of OSAHS. EDS is closely correlated to cognitive decline, anxiety, and depression. Economou et al. ${ }^{30}$ examined 36 patients, and revealed that OSAHS patients with higher ESS scores have higher Hospital Anxiety and Depression Scale (HADS) scores, and that these patients had improved ESS and HADS scores after effective continuous positive airway pressure (CPAP) therapy. In addition, other studies have observed the remission of
EDS and the relief in tension and stress of OSAHS patients after reducing their breathing disorder during sleep after OSAHS surgery ${ }^{31-34}$.

Untreated patients with severe OSAHS have more fatal and nonfatal cardiovascular events, when compared to patients with mild or moderate OSAHS ${ }^{8,35}$. Obesity and hyperglycemia would further increase adverse cardiovascular outcomes in patients with OSAHS ${ }^{36}$. Therefore, weight loss and blood glucose lowering are crucial for OSAHS adults with excessive weight and hyperglycemia, because obesity is closely associated with increased risk for $\mathrm{OSAHS}^{8,37}$, and weight loss may reduce OSAHS symptoms in addition to many other health benefits ${ }^{38}$.

CPAP and OSAHS surgery is recommended for adults with OSAHS. CPAP is the most effective treatment for OSAS, and improves the prognosis of patients with the disorder ${ }^{8}$. However, CPAP does not provide a permanent resolution ${ }^{39}$, and may not be well-tolerated by some patients, leading to poor compliance ${ }^{40}$. Surgical treatments, including uvulopalatopharyngoplasty, are associated with risks and serious adverse effects, and present evidence is limited, and is not able to demonstrate the benefits of surgery as a treatment for OSAHS $^{41}$. Finally, pharmacologic therapies have not been part of the primary treatment, and no clinically useful medication is presently listed in OSAHS treatment guidelines.

If dapagliflozin can be widely confirmed to reduce OSAHS symptoms, it may be a more acceptable treatment, when compared to CPAP or surgery. It has the potential to not only treat T2DM, but also play a significant role in patients with severe OSAHS, thereby reducing the development of cardiovascular or cerebrovascular illnesses, and the risk of death.

Limitation: Firstly, the sample size was limited and a larger sample size was needed for further study. Secondly, the influence of dapagliflozin on neck circumference and other obesity-related signs remains unknown and should be further research. Thirdly, the relationship between neck circumference and $\mathrm{AHI}, \mathrm{LSpO}_{2}, \mathrm{ESS}$ score were unclear and should be further investigated.

In conclusion, the investigators consider that dapagliflozin has therapeutic value for patients with T2DM combined with OSAHS. However, there are some limitations in the present study. (1) The study duration was short, and the cardiovascular events or deaths were not considered as the key points. Therefore, the present study was not able to determine whether dapagliflozin could improve the cardiovascular prognosis of these subjects. (2) The different effects between dapagliflozin and traditional treatment were not compared. Hence, more studies are needed in the future. On the one hand, large prospective randomized clinical trials are required to evaluate the effectiveness and safety of dapagliflozin in treating 
OSAHS, determine the additional health benefits that can be measured in comparison with other treatments, and determine whether other SGLT2 inhibitors, such as empagliflozin and canagliflozinare, can also improve the symptoms and prognosis of OSAHS patients.

\section{Acknowledgements}

The work was supported by Youth Training Program of Sichuan Provincial Science and Technology Department (No. 2016049) and Chengdu Municipal Natural Science Foundation (No. 2015-HM01-00281-SF).

\section{Author details}

'Department of Endocrinology, The Fifth People's Hospital of Chengdu, 611130 Chengdu, P.R. China. ${ }^{2}$ Center of Diabetes Mellitus, Sichuan Academy of Medical Sciences and Sichuan Provincial People's Hospital, 610072 Chengdu, P. R. China. ${ }^{3}$ Department of Respiratory, The Fifth People's Hospital of Chengdu, 611130 Chengdu, P.R. China. ${ }^{4}$ Department of Endocrinology, The Third People's Hospital of Chengdu, 610000 Chengdu, P.R. China. ${ }^{5}$ Department of Endocrinology, People's Hospital of Yilong County, 637676 Yilong, P.R. China

\section{Conflict of interest}

The authors declare that they have no conflict of interest.

\section{Publisher's note}

Springer Nature remains neutral with regard to jurisdictional claims in published maps and institutional affiliations.

Received: 9 January 2019 Revised: 23 May 2019 Accepted: 13 June 2019 Published online: 04 November 2019

\section{References}

1. Qaseem, A. et al. Clinical Guidelines Committee of the American College of Physicians. Diagnosis of obstructive sleep apnea in adults: a clinical practice guideline from theAmerican College of Physicians. Ann. Intern. Med. 161, 471-483 (2014).

2. Tuomilehto, H., Seppä, J. \& Uusitupa, M. Obesity and obstructive sleep apneaclinical significance of weight loss. Sleep Med. Rev. 17, 321 (2013).

3. Tahrani, A. A., Ali, A. \& Stevens, M. J. Obstructive sleep apnoea and diabetes: an update. Curr. Opin. Pulmon. Med. 19, 631-638 (2013).

4. Foster, G. D. et al. Sleep AHEAD Research Group. Obstructive sleep apnea among obese patients with type 2 diabetes. Diabetes Care 32, 1017-1019 (2009).

5. Weiss, J. W., Launois, S. H., Anand, A. \& Garpestad, E. Cardiovascular morbidity in obstructive sleep apnea. Prog. Cardiovasc. Dis. 41, 367-376 (1999).

6. Floras, J. S. Sleep apnea and cardiovascular risk. J. Cardiol. 63, 3-8 (2014).

7. Floras, J. S. Sleep apnea and cardiovascular disease: an enigmatic risk factor. Circ. Res. 122, 1741-1764 (2018).

8. Listed, N. Summaries for patients: diagnosis of obstructive sleep apnea in adults: a clinical practice guideline from the American College of Physicians. Ann. Intern. Med. 161, 471-U94 (2014).

9. Cal, D. R., De, V. N. \& van Wagensveld, B. A. Obstructive sleep apnea and bariatric surgical guidelines: summary and update. Curr. Opin. Anaesthesiol. 31, 104 (2017).

10. Gallo, L. A., Wright, E. M. \& Vallon, V. Probing SGLT2 as a therapeutic target for diabetes: basic physiology and consequences. Diab. Vasc. Dis. Res. 12, 78-89 (2015).

11. Merovci, A. et al. Dapagliflozin lowers plasma glucose concentration and improves $\beta$-cell function. J. Clin Endocrinol. Metab. 100, 1927-1932 (2015).

12. Hayashizaki-Someya, Y. et al. Ipragliflozin, an SGLT2 inhibitor, exhibits a prophylactic effect on hepatic steatosis and fibrosis induced by cholinedeficient I -amino acid-defined diet in rats. Eur. J. Pharmacol. 754, 19-24 (2015).

13. Gouniberthold, I., Hanssen, R., Ravarani, L. \& Berthold, H. K. Management of blood pressure and heart rate in patients with diabetes mellitus. Curr. Pharm. Design 23, 4573-4582 (2017).
14. Garber, A. J.2018 AACE T2D Algorithm Authors Consensus statement by the American Association of Clinical Endocrinologists and American College of Endocrinology on the comprehensive type 2 diabetes management algorithm-2018. Endocr. Pract. 240, 91-120 (2018).

15. Malin, S. K. \& Kashyap, S. R. Effects of metformin on weight loss: potential mechanisms. Curr. Opin. Endocrinol. Diabetes Obes. 21, 323-329 (2014).

16. Group, T. D. P. P. Long-term safety, tolerability, and weight loss associated with metformin in the Diabetes Prevention Program Outcomes Study. Diabetes Care 35, 731-737 (2012)

17. Wilding, J. et al. Changes in $\mathrm{HbA} 1 \mathrm{c}$ and weight, and treatment persistence, over the 18 months following initiation of second-line therapy in patients with type 2 diabetes: results from the United Kingdom Clinical Practice Research Datalink. BMC Med. 16, 116 (2018).

18. Kabir, A. Effect of metformin on the severity of obstructive sleep apnea in patients with diabetes mellitus (Type 2). Chest 140, 821A-821A (2011).

19. Lin, D., Rein, L., Tarima, S., Woodson, B. T., Meurer, J. R. The relationship between metformin and obstructive sleep apnea. J. Sleep Med. Disord. 2, pii: 1027 (2015)

20. Kasai, T., Bradley, T. D., Friedman, O. \& Logan, A. G. Effect of intensified diuretic therapy on overnight rostral fluid shift and obstructive sleep apnoea in patients with uncontrolled hypertension. J. Hypertension 32, 673 (2014).

21. White, L. H., Bradley, T. D. \& Logan, A. G. Pathogenesis of obstructive sleep apnoea in hypertensive patients: role of fluid retention and nocturnal rostral fluid shift. J. Hum. Hypertens. 29, 342-350 (2015).

22. Khurshid, $\mathrm{K}$. et al. Effect of antihypertensive medications on the severity of obstructive sleep apnea: a systematic review and meta-analysis. J. Clin. Sleep Med. 12, 1143-1151(2016)

23. Fiori, C. Z., Martinez, D., Gonçalves, S. C., Montanari, C. C. \& Fuchs, F. D. Effect of diuretics and sodium-restricted diet on sleep apnea severity: study protocol for a randomized controlled trial. Trials 16, 188 (2015).

24. Gaddam, K. et al. Spironolactone reduces severity of obstructive sleep apnoea in patients with resistant hypertension: a preliminary report. J. Hum. Hypertens. 24, 532 (2010).

25. Yang, L. et al. Effect of spironolactone on patients with resistant hypertension and obstructive sleep apnea. Clin. Exp. Hypertens. 38, 464-468 (2016).

26. Eskandari, D., Zou, D., Grote, L., Hoff, E. \& Hedner, J. Acetazolamide reduces blood pressure and sleep-disordered breathing in patients with hypertension and obstructive sleep apnea: a Randomized Controlled Trial. J. Clin. Sleep Med. 14, 309-317 (2018).

27. Yadollahi, A., Vena, D., Lyons, O. D. \& Bradley, T. D. Relationship of fluid accumulation in the neck to sleep structure in men during daytime sleep. J. Clin. Sleep Med. 12, 1365-1371 (2016).

28. Bolinder, J. et al. Effects of dapagliflozin on body weight, total fat mass, and regional adipose tissue distribution in patients with type 2 diabetes mellitus with inadequate glycemic control on metformin. J. Clin. Endocrinol. Metab. 97, 1020-1031 (2012)

29. Tobita, H., Sato, S., Miyake, T., Ishihara, S. \& Kinoshita, Y. Effects of dapagliflozin on body composition and liver tests in patients with nonalcoholic steatohepatitis associated with type 2 diabetes mellitus: a prospective, open-label, uncontrolled study. Curr. Ther. Res. Clin. Exp. 87, 13-19 (2017).

30. Economou, N. T. et al. Sleepiness, fatigue, anxiety and depression in chronic obstructive pulmonary disease and obstructive sleep apnea-overlap-syndrome, before and after continuous positive airways pressure therapy. PLoS One 13, e0197342 (2018).

31. Li, Y. Y. et al. Anxiety and depression are improved by continuous positive airway pressure treatments in obstructive sleep apnea. Int. J. Psychiatry Med. 51, 554 (2016).

32. Ishman, S. L. et al. Does surgery for obstructive sleep apnea improve depression and sleepiness? Laryngoscope 124, 2829-2836 (2014).

33. Gupta, M. A., Simpson, F. C. \& Lyons, D. C. The effect of treating obstructive sleep apnea with positive airway pressure on depression and other subjective symptoms: a systematic review and meta-analysis. Sleep Med. Rev. 8, 55-68 (2016).

34. Devita, M. et al. Obstructive sleep apnea and its controversial effects on cognition. J. Clin. Exp. Neuropsychol. 15, 1 (2017).

35. Geovanini, G. R. et al. Association between obstructive sleep apnea and cardiovascular risk factors: variation by age, sex, and race. The Multi-Ethnic Study of Atherosclerosis. Ann. Am. Thorac. Soc. 15, 970-977 (2018).

36. Gasa, M. et al. Obstructive sleep apnoea and metabolic impairment in severe obesity. Eur. Respir. J. 38, 1089-1097 (2011). 
37. Gohil, A. \& Hannon, T. S. Poor sleep and obesity: concurrent epidemics in adolescent youth. Front. Endocrinol. 9, 364 (2018).

38. de Assunção Machado, A. C., da Silva, A. M. V., Signori, L. U., da Costa Alvarez, G. \& Mottin, C. C. Endothelial function of patients with morbid obesity submitted to Roux-en-Y gastric bypass with and without obstructive sleep apneahypopnea syndrome. Obes. Surg. 28, 3595-3603 (2018).

39. Randerath, W. J. et al. European Respiratory Society task force on non-CPAP therapies in sleep apnoea. Non-CPAP therapies in obstructive sleep apnoea. Eur. Respir. J. 37, 1000-1028 (2011).
40. Villar, I., Izuel, M., Carrizo, S., Vicente, E. \& Marin, J. M. Medication adherence and persistence in severe obstructive sleep apnea. Sleep 32, 623-628 (2009).

41. Qaseem, A. et al. Clinical Guidelines Committee of the American College of Physicians. Clinical Guidelines Committee of the American College of Physicians. Management ofobstructive sleep apnea in adults: a clinical practice guideline from the American College of Physicians. Ann. Intern. Med. 159, 471-483 (2013) 